tion « von Rechtsideologien in rechtswissenschaftlicher Forschung und Lehre wird nur mit der Veränderung der materiellen Verhältnisse unterbrochen.

III.

Als Diskussionsgrundlage zur Politisierung der Juristen eignet sich das Loccumer Modell, insbesondere um Studienreformdebatten aus ihrer Unverbindlichkeit und Einseitigkeit herauszuführen. $\mathrm{Da}$ es das bestehende Gesellschaftssystem nicht auf seine Rationalität befragt, insgesamt gesehen, die Gewähr für systemimmanente Verwertbarkeit bietet und einen Juristen schafft, der den politischen status quo stabilisieren hilft, bleibt den jungen kritischen Juristen nur die kollektive Selbstorganisation als Lösung zur Emanzipation.

Helmut D. Fangmann

\title{
Referendarprotest in Heidelberg
}

Im Zusammenhang mit dem Heidelberger Stadthallenprozeß, bei dem s Studenten wegen ihres Vorgehens gegen ein Liederpotpourri für ausländische Studenten zur "Ordnung" gerufen wurden (Ordnung hieß in diesem Fall Gefängnis), kam es am 10. Januar 1969 zu einer spektakulären Polizeiaktion in den Räumen des Heidelberger AStA anläßlich der Vollstredkung des Haftbefehls gegen die Fünf. Durch Presse, Rundfunk und Fernsehen wurden die Ausschreitungen der Polizei bekannt. Wer geglaubt hatte, den politischen Studenten mit der Aktion das Genick brechen zu können, sah sich getäuscht. Die Studentenbewegung wurde stärker, und der Funke sprang gleichzeitig auf die Gerichtsreferendare über. I7 Referendare forderten die Einleirung eines Disziplinar- und Strafverfahrens gegen den am Einsatz beteiligten Staatsanwalt Weidner. Versuche des reaktionären Oberstaatsanwalts Nägele, die Referendare mit Hilfe eines Disziplinarverfahrens zum Schweigen zu bringen, führten zu einem Aufbegehren der Lokalpresse und zu einer Solidarisierung weiterer Referendare. Der Heidelberger Landgerichtspräsident Kohnle konnte sich einer Diskussion mit den Gerichtsreferendaren über die Vorfälle nicht entziehen; der Fall zog weitere Kreise.

Es konstituierte sich nun eine Arbeitsgruppe Heidelberger Referendare, und nachdem im Juni 1969 ein progressiver Vorstand für den Bezirksreferendarverband Nordbaden gewählt war, konnte man mit gezielten Aktionen Aufklärungsarbeit in der Offentlichkeit leisten. Nachdem die Anwaltskammer Nordbaden in drei skandalösen Gutachten der Zulassung der Assessoren Jürgen Laubscher, Marlies und Eberhard Bedker zum Anwalesberuf widersprochen hatte, machten die Referendare durch eine Flublattalstion und eine scharfe in der Presse veröffentlichte Resolution den Weg für eine Diskussion in der Mitgliederversammlung der Anwaltskammer frei. Sie forderten, bei der Diskussion über die Gutachten die Offentlichkeit herzustellen, zumindest aber eine Delegation der Gerichtsreferendare als Vertreter der Interessen der Betroffenen zuzulassen. Einem vordemokratischen Standesdenken verhafter, lehnten die Anwälte diese 
Forderung ab, so daß auch die in großer Anzahl erschienenen Journalisten wieder abziehen mußten. Die Presse nahm sich der Vorfälle an, und weitere bedenkliche Entscheidungen der Kammer konnten aufgedeckt werden. In einigen Fällen war den "gemaßregelten « Anwälten der primitivste Schutz des rechtlichen Gehörs verweigert worden. Auch unter den Anwälten setzte ein gewisser Bewußtwerdungsprozeß ein, sie starteten eine Kampagne gegen die autoritären $»$ Kammerherren ", der Vorstand des Heidelberger Anwaltsvercins wurde abgewählt. Die Referendare konnten in einem Fernsehinterview eine breite Ofrentlichkeit über die Handlungsweise der Anwaltskammer aufklären.

Auf dem die Referendare unmittelbar interessierenden Ausbildungssektor gelang es, cinen Richter, der sich durch besonders autoritäre Verhandlungsï̈ürung, u. a. in einem Studentenprozeß, "ausgezeichnet " hatte, von der Referendarausbildung abzulösen, nachdem die Referendare in einem Zeitungsartikel seine Verhandlungsführung analysiert und kritisiert hatten.

Die Arbeitsgruppe erstellte einen Entwurf zur Reform der juristiscren Ausbildung (abgedrudkt in "Recht und Politik « 3, 1969, S. I 1 o), der in einer vom OLG Karlsruhe veranstalteten Arbeitswoche für Gerichtsreferendare in Steinbach diskutiert werden sollte. Gleich zu Anfang der Arbeitswoche nahmen die Teilnehmer, angeregt durch den Referendarverband Nordbaden, kritisch zu dem vom OLG ausgearbeiteten Programm der Arbeitswoche Stellung und stimmten mit großer Mehrheit für ein vom Referendarverband Nordbaden angeborenes und mehr an gesellsclaattspolitischen Problemen und der Ausbildungsreform ausgerichtetes Alternativprogramm. In der sich nun anschließenden Auseinandersetzung mit dem OLG-Präsidenten zeigte sich jedoch, daß die spontan zustandegebrachte Solidarität noch nicht durch ein ausreichendes Bewußtsein abgesichert war, um in der Konfrontation mir der Autorität, hinter der das Disziplinarrecht lauert, bestehen zu können. Das OLG konnte dann schließlich doch mit dem Einverständnis der Mehrheit der Referendare sein ursprüngliches Programm durchführen. $\mathrm{DaB}$ aber durch den Konflikt dennoch ein nicht zu unterschätzender Lernprozeß in Gang gebracht wurde und konkret erreicht wurde, daß die künftigen Arbeitswochen von Referendaren mitgestaltet werden sollen, ist als wesentlicher Erfolg anzusehen. In der Diskussion über die Ausbildungsreform wurden dem baden-württembergischen Justizminister Schieler die Forderungen der Referendare, die in praktisch allen Punkten mit dem "Heidelberger Entwurf « übereinstimmten, vorgelegt. Seine Zustimmung erhielten jedoch nur die Punkte, die mehr oder weniger vage langfristige Zukunftsperspektiven enthalten (auf deren Mitgestaltung der Minister selbst kaum Einfluß hat), oder Punkte, die eine technisch perfektere Ausgestaltung des bisherigen Ausbildungssystems zum Gegenstand haben. Auf taube Ohren stießen dagegen die Forderungen nach Mitbestimmung und Demokratisierung der Ausbildung (z. B. die Möglicikeit der Abwahl von Arbeirsgemeinschaftsleitern), Abschaffung des Assessorexamens und wesentlicher Verkürzung und Strafiung des Vorbereitungsdienstes. Daher wurde die am 13. November durchgeführte Demonstration der Gerichtsreferendare in Stuttgart genau auf die Ebene getragen, auf der sich in Steinbach die fundamentalen Gegensätze gezeigt haben und die Irrationalität der Ausbildungspolitik verdeutlicht wurde. Uber Unterhaltszuscheßerhöhungen oder Abschriften von Stationszeugnissen nämlich sind dic Vertrecer der Regierung vergle:chsweise gern bereir mit uns zu diskuticren und Lösungen zu finden, denn da handelt es sich um technische Fragen, die die traditionellen Strukturen unangetaster lassen. Die Auseinandersetzung muß durch noch stärkere Offentlichkeitsarbeit vorbereiter und unterstützt werden. 
Hicrbei spielt auch eine wesentliche Rolle, daß die Auseinandersetzung mit Institutionen und Strukturen auf eine immer breiter werdende solidarische Basis gestellt werden muß, die sich über die Grenzen des juristischen Bereichs hinaus auf andere Gruppen erstreckt. In Heidelberg wurde jetzt mit einer Zusammenarbeit von Gerichtsreferendaren und Studienreferendaren der Anfang gemacht. Ein konkreter Anlaß gab den Anstoß hierzu: dem früheren AStA-Vorsitzenden Steffens wurde die Zulassung als Studienreferendar vom Oberschulamt Nordbaden mit einer ähnlichen Begründung abgelehnt wie in den oben erwähnten Fällen der drei Rechtsassessoren. Gemeinsame Teach-ins, Resolutionen und Pressekonferenzen, eine Demonstration dienen dazu, spontane solidarische Aktionen in festere Organisationsformen übergehen zu lassen, als Folge des Bewußtseins der vergleichbaren Situation der beiden Gruppen im Apparat und gegenüber dem traditionellen Beamtenrecht.

Wichtig ist, daß auch die vertikale Verbindung zu den studentischen Institutsgruppen verstärkt wird und der Eintritt des engagierten Studenten in das besondere Gewaltverhältnis des Beamten auf Widerruf sich dank der vertikalen Kontinuität der kritischen Juristenbewegung ohne zu großen Bruch vollzieht. Die praktischen beruflichen Erfahrungen der Gerichtsreferendare werden den juristischen Institutsgruppen in deren Auseinandersetzungen mit der Hochschule theoretisch und praktisch von Nutzen sein.

Arbeitsgruppe Heidelberger Gerichtsreferendare

\section{Referendarwiderstand: Beispiel Hamburg}

Anfang Februar dieses Jahres fand im Hanseatischen OLG in Hamburg eine ungewöhnliche Vollversammlung der hiesigen Referendare statt. Die bis dahin friedlichen und eher als konservativ geltenden Hamburger Referendare wollten nicht länger die seit nahezu roo Jahren ohne wesentliche Anderungen praktizierte Ausbildung hinnehmen. Mit überwältigender Mehrheit wurde beschlossen, daß der Hamburger Referendarausschu $\beta$ - die Vertretung der Hamburger Referendare - in Zukunft nur noch für die Abschaffung des gegenrwärtigen, dualistischen Ausbildungssystems zu sorgen habe. Um dem Ausschuß einen gewissen Spielraum zu lassen, wurde ihm gestatter, bis zur Erreichung des grundsätzlichen Ziels, auf die Abschaffung des 2. Staatsexamens zu dringen, bzw. auf eine grundlegende Veränderung dieses 2. Staatsexamens.

Dieses Ereignis wäre an sich nicht besonders erwähnenswert, denn die Unzufriedenheit der Referendare mit der derzeitigen Ausbildung ist wohl in ganz Deutschland die gleichet. Erwähnenswert ist das Bemühen der Hamburger Referendare nur, weil sie als erste in Deutschland gezeigt haben, daß sie nicht gewillt sind, sich von leeren Reformversprechen und den üblichen Manövern der für die derzeitige Ausbildung Verantwortlichen täuschen zu lassen.

Nachdem der Justizsenator und die maßgeblichen Herren des OLG erkannt hatten, daß bloße Reformversprechen nicht genügen würden, die unruhigen Re-

I Siehe auch die Berichte von Referendargruppen aus Berlin, Frankfurt und Heidelberg, Kritische Justiz 1969, S. 181, S. 292 und S. 411. 